\title{
CIRUGÍA REVISIONAL POR ESTENOSIS POST GASTRECTOMÍA VERTICAL LAPAROSCÓPICA*
}

\author{
Drs. Ana María Burgos L. ${ }^{1}$, Attila Csendes J. ${ }^{1}$, Italo Braghetto M. ${ }^{1}$, Andrea Muñoz CH. ${ }^{1}$ \\ 1 Departamento de Cirugía, Hospital Clínico de la Universidad de Chile. \\ Santiago, Chile.
}

\begin{abstract}
Revisional surgery for stenosis following laparoscopic sleeve gastrectomy

Introduction: Revisional bariatric surgery is performed to alter or repair one of the many types of weight loss surgery, including laparoscopic sleeve gastrectomy (LSG). The revision of bariatric procedures is required between 10 to $25 \%$ of patients operated, either by insufficient weight loss or complications, including stenosis. After a LSG, the Roux- en- Y gastric bypass (RYGBP) is the most requested surgery for conversion. The aim of this study was to describe a series of 5 patients who had revisional surgery for stenosis following LSG. Material and Method: Between January 2006 and March 2013, in 770 consecutive patients was performed LSG. Patients were included in a prospective protocol. Five patients had revisional surgery with conversion to RYGBP for stenosis following LSG. In three patients the initial LSG was performed in our hospital, the other 2 were from other hospitals. Results: In the 5 patients with revisional surgery mean age was $39.8 \pm 15.7$ years. Four patients were women (80\%). The average BMI before LSG was $37.0 \pm 2.1$ $\mathrm{kg} / \mathrm{m}^{2}$ and BMI before RYGBP was $29.4 \pm 5.4 \mathrm{~kg} / \mathrm{m}^{2}$. The RYGBP was laparoscopic in 3 cases and opened in two. There were no complications, and no mortality. Conclusions: Conversion to RYGBP is an effective treatment for stenosis following a LSG.
\end{abstract}

Key words: Revisional surgery, morbid obesity, vertical gastrectomy, gastric bypass, stenosis.

\section{Resumen}

Introducción: La cirugía bariátrica revisional se realiza para modificar o reparar alguno de los tipos de cirugía para perder peso, entre ellos la gastrectomía vertical laparoscópica (GVL). La revisión de procedimientos bariátricos se hace necesaria entre el $10 \mathrm{al} 25 \%$ de los pacientes, ya sea por baja de peso insuficiente o por complicaciones, siendo la estenosis una de ellas. Posterior a una GVL, el bypass gástrico en Y de Roux (BPG) es la intervención quirúrgica más requerida para la conversión. El objetivo de este estudio fue describir una serie de 5 pacientes que tuvieron cirugía revisional por estenosis posterior a una GVL. Material y Métodos: Entre enero de 2006 y marzo de 2013, en 770 pacientes consecutivos se realizó GVL. Los pacientes fueron incluidos en un protocolo prospectivo. Cinco pacientes tuvieron cirugía revisional con conversión a BPG por

*Recibido el 6 de mayo de 2013 y aceptado para publicación el 24 de junio de 2013.

Los autores no refieren conflictos de interés.

Correspondencia: Dra. Ana María Burgos L. Santos Dumont 999, Santiago, Chile. anamburgos@yahoo.com.mx 
estenosis posterior a la GVL. Tres pacientes fueron operados de GVL en nuestro hospital, los otros 2 provenían de otros centros hospitalarios. Resultados: En los 5 pacientes con cirugía revisional la edad promedio fue 39,8 $\pm 15,7$ años. Cuatro pacientes fueron mujeres (80\%). El promedio IMC antes de GVL fue 37,0 $\pm 2,1$ $\mathrm{kg} / \mathrm{m}^{2}$ y del IMC antes del BPG fue $29,4 \pm 5,4 \mathrm{~kg} / \mathrm{m}^{2}$. El BPG fue laparoscópico en 3 casos y abierto en 2 . No hubo complicaciones, ni mortalidad. Conclusiones: La conversión a bypass gástrico en $\mathrm{Y}$ de Roux es un tratamiento efectivo para la estenosis posterior a una GVL.

Palabras clave: Cirugía revisional, obesidad mórbida, gastrectomía vertical, bypass gástrico, estenosis.

\section{Introducción}

La cirugía bariátrica revisional se realiza para modificar o reparar uno de los muchos tipos de cirugía para perder peso, entre ellos la GVL ${ }^{1}$. La GVL ha surgido como una técnica quirúrgica restrictiva reproducible y efectiva para la baja de peso con regresión de las comorbilidades asociadas, ya que reduce los niveles de la hormona orexígena grhelina y estimula la producción de incretinas por vaciamiento acelerado del tubo gástrico, reportándose una reducción entre el 33 y $66 \%$ del exceso de peso ${ }^{2}$.

La revisión de procedimientos bariátricos se requiere entre 10 a $25 \%$ de los pacientes, ya sea por baja de peso insuficiente o por complicaciones ${ }^{3,4}$. Entre las complicaciones que se pueden presentar después de una GVL, una es la estenosis, existiendo para ella diversos tratamientos como dilataciones endoscópicas, endoprótesis gastrointestinales, plastía de las estrecheces y la cirugía revisional, siendo el BPG la cirugía más requerida para su solución. El objetivo de este estudio fue describir una serie de 5 pacientes que tuvieron cirugía revisional por estenosis posterior a una GVL.

\section{Pacientes y Métodos}

Entre enero de 2006 a marzo de 2013, en 770 pacientes consecutivos se realizó GVL. Todos los pacientes fueron incluidos en un protocolo prospectivo. Un total de 5 pacientes tuvieron cirugía revisional con conversión a BPG por presencia de estenosis posterior a la GVL. Tres pacientes fueron operados de GVL inicialmente en nuestro departamento y los otros 2 provenían de otros centros hospitalarios.

La GVL estuvo indicada en los pacientes según las normas establecidas por el National Institute of Health (NIH) de Estados Unidos de Norteamérica ${ }^{5}$, que incluye pacientes con un Índice de Masa Corporal (IMC) de $35 \mathrm{~kg} / \mathrm{m}^{2}$ y comorbilidades asociadas, o un IMC de $40 \mathrm{~kg} / \mathrm{m}^{2}$ en que ha fallado el tratamiento médico. Los criterios de exclusión fueron la presencia de esofagitis severa, esófago de Barrett, hernia hiatal mayor a $5 \mathrm{~cm}$, así como presencia de alcoholismo, drogadicción o enfermedades psiquiátricas.

Posterior a la GVL, en todos los pacientes al $3^{\circ}$ día postoperatorio (DPO) se realizó, por médicos del Departamento de Radiología, radiografía gastrointestinal con contraste líquido de sulfato de bario, evaluándose las características del estómago tubular, vaciamiento, presencia de estenosis y filtraciones.

\section{Técnica quirúrgica}

La GVL fue realizada según técnica ampliamente descrita en publicaciones previas ${ }^{6,7}$, uso de cinco trócares y sonda 37-F a través del lumen gástrico, para evitar estrechez del estómago. Inicio de la sección gástrica 2-3 cm del píloro y hasta $1 \mathrm{~cm}$ antes del ángulo de His empleando sutura mecánica y formando un estómago tubular con capacidad entre 60 a $80 \mathrm{ml}$.

La conversión de la GVL a BPG laparoscópico es similar al BPG primario usando 5 trócares, sección proximal a la zona de estenosis con engrapadora lineal cortante (carga azul) y anastomosis entre estómago proximal dilatado y asa yeyunal ascendida, previa identificación del ángulo de Treitz y ascenso de asa yeyunal de $80 \mathrm{~cm}$ aproximadamente, antecólica. Se asciende asa yeyunal (asa alimentaria) de 150 a $100 \mathrm{~cm}$, dependiendo del IMC que presente el paciente, y entero-entero anastomosis con sutura mecánica contigua a anastomosis previa. Prueba de hermeticidad con azul de metileno. Sección yeyunal entre anastomosis quedando conformada la $\mathrm{Y}$ de Roux.

\section{Estadística}

El registro de los datos y análisis estadístico se realizó con el programa estadístico SPSS 16,0 (Chicago, IL, USA). Los datos se expresaron como promedios, desviación estándar y rango.

\section{Casos reportados}

\section{Caso 1}

Varón de 34 años de edad, con IMC de 39,7 kg/ $\mathrm{m}^{2}$ con hipertensión arterial y dislipidemia mixta, es sometido a GVL. La cirugía se realizó según técnica habitual del Servicio de Cirugía, usándose sonda 37 F para calibración del tubo gástrico y sin refuerzo de la sutura mecánica. El día siguiente presentó náuseas, vómitos, regurgitación y sialorrea, al $2^{\circ}$ día una radiografía de esófago, estómago y duo- 
deno (EED) evidencia pasaje lento y filiforme del contraste administrado vía oral. Al $3^{\circ}$ día inicia régimen hídrico oral y después líquido, siendo dado de alta al $4^{\circ}$ día. Continúa con regurgitación y vómitos, al $9^{\text {a }}$ día presenta fiebre de $38,5^{\circ} \mathrm{C}$. Reingresa al hospital al $10^{\circ}$ día con deshidratación e insuficiencia aguda pre-renal; una endoscopía digestiva alta (EDA) muestra estenosis en cuerpo gástrico, instalándose sonda nasoyeyunal para alimentación, al $21^{\circ}$ día posoperatorio (DPO) se hace dilatación endoscópica, pasando el endoscopio de $15 \mathrm{~mm}$ de diamétro. La radiografía de EED muestra paso lento contraste hidrosoluble a través del esófago y fondo gástrico dilatado, y a nivel del ángulo gástrico hay una pérdida abrupta de calibre de $2,5 \mathrm{~cm}$ de longitud (Figura 1). Al 27 DPO se realiza una conversión a bypass gástrico en $\mathrm{Y}$ de Roux no resectivo por vía laparoscópica, evoluciona satisfactoriamente con ingesta oral adecuada y se da el alta al $5^{\circ}$ día posterior a esta cirugía.

\section{Caso 2}

Mujer de 32 años con IMC de $24,7 \mathrm{~kg} / \mathrm{m}^{2}$ operada de GVL 3 años antes en otro Centro, con reoperaciones múltiples por fístula. Posteriormente, evoluciona con regurgitación, disfagia a sólidos y tolerando sólo papilla. La radiografía de EED y EDA evidencian estenosis en parte alta del tubo gástrico y esofagitis severa, realizándose conversión a bypass gástrico en Y de Roux con resección del segmento estenótico vía abierta por las múltiples laparotomías y hernia incisional. En la cirugía se liberan adherencias de pared anterior gástrica y se realiza anastomosis gastroyeyunal manual usando Byosin ${ }^{\circledR}$ 3-0, entre estómago proximal dilatado y yeyuno distal seccionado a $70 \mathrm{~cm}$ del ángulo de Treitz, completando con anastomosis yeyuno-yeyunal termino-lateral manual y drenaje abdominal (Figura 2). La paciente evoluciona en óptimas condiciones y es dada de alta al $6^{\circ}$ $\mathrm{DPO}$, tolerando bien la ingesta oral.

\section{Caso 3}

Mujer de 65 años, con IMC de $36,2 \mathrm{~kg} / \mathrm{m}^{2}$, resistencia a la insulina, hernia hiatal gigante grado III y anemia crónica. Se realiza reducción de hernia hiatal, fundoplicatura de Nissen y GVL. Posteriormente, presenta disfagia a alimentos sólidos, náuseas y dolor retroesternal. La EDA muestra esófago con abundante reflujo biliar. Se realiza conversión a bypass gástrico con resección gástrica distal por vía abierta, por múltiples cirugías previas y hernia incisional. En la cirugía se observan adherencias del

estómago a la cara inferior del hígado y una estenosis mesogástrica (Figura 3), se realiza sección proximal a la estenosis y distal a nivel duodenal con extirpación del estómago distal. Se realiza anastomosis gastro-yeyunal retrocólica y yeyuno-yeyunal con Covidien`s Endo GIA ATM $^{\mathrm{O}}$ morado. Presenta evolución satisfactoria, con
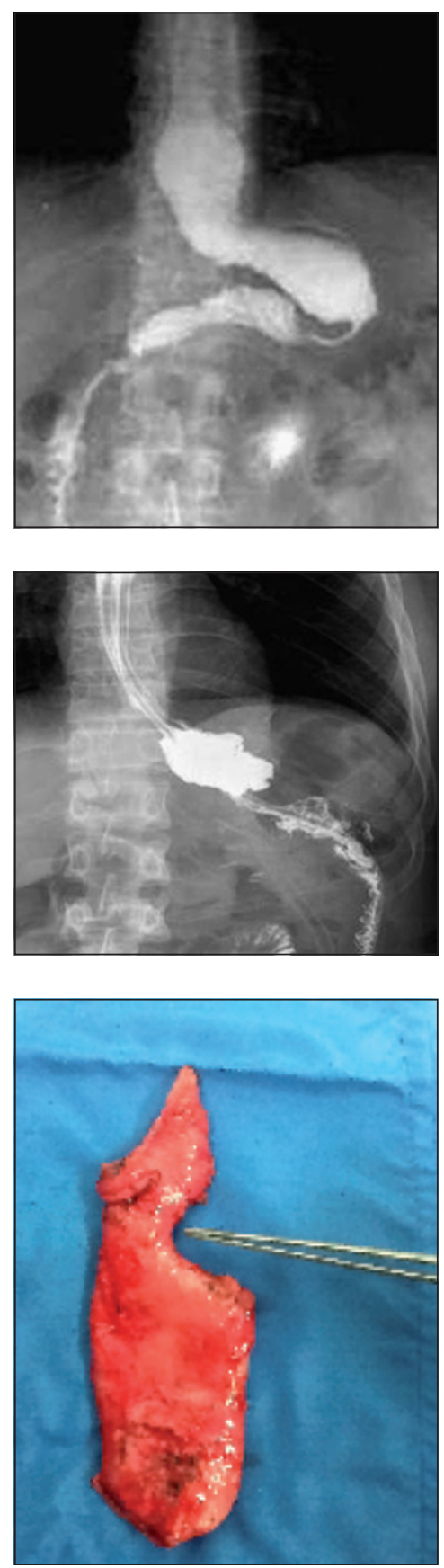

Figura 1. Estenosis a nivel de tercio medio en estómago tubular post GVL.

Figura 2. Conversión a bypass gástrico con Y de Roux.
Figura 3. Estómago distal con zona de estenosis, resecado durante bypass gástrico vía abierta. 
Tabla 1. Datos demográficos de 5 pacientes con cirugía revisional por estenosis posterior a GVL

\begin{tabular}{|c|c|c|c|c|c|}
\hline Parámetros & Caso 1 & Caso 2* & Caso 3 & Caso 4* & Caso 5 \\
\hline Edad (años) & 34 & 32 & 65 & 44 & 24 \\
\hline Género & Masculino & Femenino & Femenino & Femenino & Femenino \\
\hline $\begin{array}{l}\text { IMC antes GVL } \\
\left(\mathrm{kg} / \mathrm{m}^{2}\right)\end{array}$ & 39,7 & 39,0 & 36,2 & 35,0 & 35,5 \\
\hline $\begin{array}{l}\text { IMC antes BPG } \\
\left(\mathrm{kg} / \mathrm{m}^{2}\right)\end{array}$ & 37,5 & 24,7 & 29,8 & 24,1 & 30,9 \\
\hline $\begin{array}{l}\text { Tiempo entre } \\
\text { GVL y BPG }\end{array}$ & 27 días & 3 años & 3 meses & 3 años & 4 años \\
\hline Co-morbilidades & $\begin{array}{c}\text { Hipertensión } \\
\text { arterial } \\
\text { Dislipidemia } \\
\text { Esteatosis hepática }\end{array}$ & $\begin{array}{l}\text { Resistencia a la } \\
\text { insulina }\end{array}$ & $\begin{array}{l}\text { Resistencia a la } \\
\text { insulina } \\
\text { Hernia hiatal } \\
\text { gigante } \\
\text { Anemia crónica }\end{array}$ & $\begin{array}{l}\text { Hernia discal } \\
\text { lumbar }\end{array}$ & $\begin{array}{c}\text { Resistencia a la } \\
\text { insulina } \\
\text { Dislipidemia } \\
\text { Hernia discal } \\
\text { lumbar } \\
\text { Esteatosis hepática }\end{array}$ \\
\hline
\end{tabular}

GVL: Gastrectomía vertical laparoscópica. BPG: Bypass gástrico en Y de Roux. *GVL realizada en otro centro.

alimentación oral adecuada y es dada de alta al $4^{\circ}$ DPO.

\section{Caso 4}

Paciente mujer de 44 años, con IMC de 24,1 kg/ $\mathrm{m}^{2}$, quien 3 años antes en otro Centro, fue operada de GVL, en esa oportunidad con IMC preoperatorio de $34 \mathrm{~kg} / \mathrm{m}^{2}$. Inmediatamente posterior a la GVL presentó vómitos frecuentes y epigastralgia, tolerando sólo papilla hasta el momento que consulta por esta sintomatología. Una EDA muestra esofagitis erosiva y un fondo gástrico dilatado. Se realiza conversión a bypass gástrico sin resección gástrica por vía laparoscópica con sección proximal a la estenosis gástrica y anastomosis mecánica entre estómago proximal dilatado y el asa yeyunal ascendida antecólica, asa alimentaria de $80 \mathrm{~cm}$. Presenta buena evolución y es dada de alta al $4^{\circ}$ DPO.

\section{Caso 5}

Paciente mujer de 24 años, con IMC de $35,5 \mathrm{~kg} /$ $\mathrm{m}^{2}$ resistencia a la insulina, dislipidemia, discopatía lumbar e hígado graso. Se le realiza una GVL evolucionando en el postoperatorio inmediato sin complicaciones. La paciente no acude a controles posteriormente. Cuatro años después consulta por dolor abdominal y vómitos postprandiales con un IMC de $30,9 \mathrm{~kg} / \mathrm{m}^{2}$. Se le realizan exámenes imagenológicos y EDA evidenciándose una estenosis mesogástrica y presencia de colelitiasis. Se le realiza una conversión a bypass gástrico con resección gástrica distal y colecistectomía laparoscópica con resolución de sus síntomas.

\section{Resultados}

De los 5 pacientes a los que se realizó cirugía revisional, 3 de ellos habían sido operados inicialmente en nuestro hospital, correspondiendo al 0,38\% de las 770 cirugías de GVL realizadas. En los 5 pacientes con cirugía revisional la edad promedio fue 39,8 $\pm 15,7$ años (rango 24-65). Cuatro pacientes fueron mujeres $(80 \%)$. El promedio de IMC antes de la GVL fue $37,0 \pm 2,1 \mathrm{~kg} / \mathrm{m}^{2}$ (rango $35,0-39,7$ ) y del IMC antes del BPG fue $29,4 \pm 5,4 \mathrm{~kg} / \mathrm{m}^{2}$ (rango 24,1-37,5) (Tabla 1).

El BPG en 3 casos fue laparoscópico, 2 sin resección y 1 con resección gástrica distal realizándose la sección duodenal con engrapadora lineal cortante (carga azul).

El BPG fue abierto en 2 casos, en 1 paciente con resección del segmento estenótico gástrico y anastomosis manual, en el otro paciente resección gástrica distal incluyendo la zona estenótica con anastomosis gastroyeyunal manual y yeyuno-yeyunal mecánica, en ambos casos con ascenso del asa yeyunal retrocólica (Tabla 2). No hubo complicaciones ni mortalidad.

\section{Discusión}

La cirugía revisional bariátrica se ha definido como la reintervención realizada posterior a una cirugía bariátrica primaria debido al fracaso de la técnica por causas diversas. El bypass gástrico en Y de Roux es considerado el procedimiento de elección 
Tabla 2. Características clínicas y cirugía revisional en 5 pacientes con estenosis post GVL

\begin{tabular}{|llllll|}
\hline & Caso 1 & Caso 2* & Caso 3 & Caso 4* & Caso 5 \\
$\begin{array}{l}\text { Sintomatología } \\
\text { post GVL }\end{array}$ & $\begin{array}{l}\text { Disfagia a líquidos } \\
\text { Regurgitación } \\
\text { Vómitos }\end{array}$ & $\begin{array}{l}\text { Disfagia a sólidos } \\
\text { Regurgitación }\end{array}$ & $\begin{array}{l}\text { Disfagia a sólidos } \\
\text { Náuseas } \\
\text { Dolor retro- } \\
\text { esternal }\end{array}$ & $\begin{array}{l}\text { Disfagia a sólidos } \\
\text { Náuseas } \\
\text { Epigastralgia }\end{array}$ & $\begin{array}{l}\text { Vómitos } \\
\text { Dolor abdominal }\end{array}$ \\
$\begin{array}{l}\text { Tipo cirugía } \\
\text { revisional }\end{array}$ & $\begin{array}{l}\text { BPG sin resección } \\
\text { gástrica distal }\end{array}$ & $\begin{array}{l}\text { BPG con resección } \\
\text { segmento este- } \\
\text { nótico }\end{array}$ & $\begin{array}{l}\text { BPG con resección } \\
\text { gástrica distal }\end{array}$ & $\begin{array}{l}\text { BPG sin resección } \\
\text { gástrica distal }\end{array}$ & $\begin{array}{l}\text { BPG con resección } \\
\text { gástrica distal } \\
\text { Colecistectomía }\end{array}$ \\
$\begin{array}{l}\text { Vía abordaje } \\
\text { BPG }\end{array}$ & Laparoscópica & Abierta & Abierta & Laparoscópica & Laparoscópica \\
\hline
\end{tabular}

GVL Gastrectomía vertical laparoscópica, BPG Bypass gástrico en Y de Roux. *GVL realizada en otro centro.

para la revisión de una GVL. De 5 cirugías revisionales realizadas, en $3(0,38 \%)$ la cirugía inicial de GVL había sido realizada en nuestro hospital en un total de 770 pacientes. La literatura menciona cifras entre 5,5 y $11 \%$ de pacientes que requieren revisión, por esta y otras causas, después de una $\mathrm{GVL}^{8}$.

Las indicaciones comunes para la revisión de la GVL son pérdida inadecuada de peso o reganancia de éste luego de una pérdida adecuada inicial, causada por cambios adaptativos como aumento en capacidad del estómago tubular; otra indicación es por complicaciones como filtraciones, sangrado, reflujo gastroesofágico severo y estenosis del estómago tubular, entre otras. La presencia de reflujo gastroesofágico (RGE) posterior a GVL es algo complicado de explicar, algunos autores reportan que éste aumenta debido a modificaciones de la anatomía esofagogástrica ${ }^{9,10}$, en tanto que otros postulan que el reflujo disminuye ${ }^{11,12}$, no habiendo consenso al respecto ${ }^{13}$.

La estenosis posterior a una GVL, es una complicación reportada con frecuencia que varía entre $0,1 \%$ y $3,9 \% 0^{14,15}$. La estenosis puede ser tratada con dilatación endoscópica con bujía o balón ${ }^{16}$, también se ha descrito el uso de stents ${ }^{17}$; en relación al tratamiento quirúrgico se describen algunos procedimientos como la seromiotomía ${ }^{18}$, plastía de la estrechez ${ }^{19}$ y la conversión a bypass gástrico ${ }^{20}$.

Algunos pacientes pueden presentar mejoría de la estenosis con dilataciones endoscópicas con balón, pero en algunos casos puede persistir sintomatología como disfagia y regurgitación siendo necesario recurrir a la cirugía revisional con una conversión a bypass gástrico en Y de Roux. Lacy $^{20}$, en su serie de 294 pacientes obesos operados con GVL reporta estenosis en 3 de ellos $(1,02 \%)$, una de ellas localizada en la unión gastroesofágica y las otras 2 con localización central, mencionando que el paciente con estenosis alta requirió dilatación endoscópica y las otras 2 se resolvieron por una conversión a bypass gástrico seccionando el estómago proximal a la estenosis, proponiendo que el tratamiento quirúrgico debe ser considerado cuando todos los otros procedimientos fallan.

Antes de realizar la cirugía revisional es necesario tener una evaluación completa preoperatoria para explicar la anatomía gastrointestinal del paciente. Los estudios radiológicos contrastados del tubo digestivo alto definen la ubicación e integridad de la línea de corchetes gástricos, así como la anatomía, tracto de salida y vaciamiento del estómago. En un estudio Triantafyllidis y cols ${ }^{21}$, posterior a una GVL, identifican 3 patrones radiológicos gástricos: el tubular, con bolsa proximal y distal. Describen en 2 pacientes estenosis y dilatación esofágica distal con reflujo del medio de contraste en posición de pie.

Después de la cirugía primaria, ocasionalmente la visión de la anatomía gástrica ya sea por endoscopía y/o radiología puede ser difícil de interpretar, y no resulta tan obvia hasta que se realiza la reintervención quirúrgica. Esto lo pudimos evidenciar en nuestro tercer paciente, en que, previo a la reintervención, la EDA mostraba pasaje del endoscopio a través del lumen gástrico y la radiografía contrastada no mostraba zonas de estrechez a pesar que la paciente clínicamente era sintomática y en el segmento gástrico resecado esta estenosis fue evidente (Figura 3).

Los resultados funcionales de la conversión de la GVL en BPG en series reportadas son excelentes en términos de baja de peso y mejoría de las comorbilidades.

Existen estudios en reoperaciones de cirugía bariátrica que muestran tasas de morbilidad perioperatoria que van desde el $10 \%$ hasta el $50 \%$, y cifras de mortalidad de hasta el $2 \%{ }^{22}$. Aunque el BPG es la solución ideal para una GVL fallida, el riesgo de las complicaciones severas postoperatorias hace que la indicación deba ser llevada a cabo por cirujanos con experiencia en cirugía bariátrica y laparoscópi- 
ca avanzada, en centros con alto volumen ${ }^{23,24}$. Una de las dificultades encontradas al momento de una reoperación es la presencia de adherencias entre los órganos comprometidos.

En nuestro estudio 3 cirugías fueron realizadas laparoscópicamente. La literatura menciona que el abordaje laparoscópico de la cirugía revisional bariátrica ha demostrado ser seguro, con tasas de morbilidad comparables a la cirugía abierta. La visión laparoscópica permite una mejor visión y una disección más exacta. En los 2 pacientes que fueron abordados por vía abierta se habían realizado previamente extensas y múltiples cirugías abiertas.

Técnicamente la conversión de GVL a BPG implica la sección proximal del remanente gástrico tubular para crear el reservorio gástrico, pudiendo el segmento gástrico tubular distal ser dejado in situ o extirpado. En la conversión posterior a una estenosis generalmente la presencia de un estómago proximal dilatado facilita la elaboración de la anastomosis gastroyeyunal. La anastomosis gastroyeyunal en nuestros 2 casos abordados laparoscópicamente fue mecánica y en los 2 por vía abierta una mecánica y la otra manual.

En conclusión, este reporte muestra la factibilidad de conversión de una GVL a BPG ya sea por vía laparoscópica o laparotómica con buenos resultados.

\section{Referencias}

1. Zundel N, Hernández J. Revisional surgery after restrictive procedures for morbid obesity. Surg Laparosc Endosc Percutan Tech. 2010;5:338-43.

2. Mechanick JI, Kushner RF, Sugerman HJ, GonzálezCampoy JM, Collazo-Clavell ML, Spitz AF, et al. American Association of Clinical Endocrinologists, The Obesity Society, and American Society for Metabolic \& Bariatric Surgery Medical Guidelines for Clinical Practice for the Perioperative Nutritional, Metabolic, and Nonsurgical Support of the Bariatric Surgery Patient. Surg Obes Relat Dis. 2008;S109-84.

3. Buckwalter JA, Herbst CA, Khouri RK. Morbid obesity second gastric operations for poor weight loss. Am Surg. 1985;51:208-11.

4. Fakhry SA, Herbst CA, Buckwalter JA. Complications requiring operative intervention after gastric bariatric surgery. South Med J. 1985;78:536-8.

5. NIH Consensus Statement. Gastrointestinal surgery for severe obesity. 1991;279:1-20.

6. Burgos AM, Braghetto I, Csendes A, Maluenda F, Korn $\mathrm{O}$, Yarmuch J, et al. Gastric leak after laparoscopic sleeve gastrectomy for obesity. Obes Surg. 2009;19:1672-7.

7. Csendes A, Braghetto I, León P, Burgos AM. Management of leaks after laparoscopic sleeve gastrectomy in patients with obesity. J Gastrointest Surg. 2010;
14:1343-48.

8. Van Rutte PW, Smulders J, de Zoete J, Nienhuijs S. Indications and short-term outcomes of revisional surgery after failed or complicated sleeve gastrectomy. Obes Surg. 2012;22:1903-8.

9. Braghetto I, Lanzarini E, Korn O, Valladares H, Molina JC, Henríquez A. Manometric changes of the lower esophageal sphincter after sleeve gastrectomy in obese patients. Obes Surg. 2010;20:357-62.

10. Braghetto I, Csendes A, Korn O, Valladares H, González P, Henriquez A. Gastroesophageal reflux disease after sleeve gastrectomy. Surg Laparosc Endosc Percutan Tech. 2010;20:148-53.

11. Santoro S. Technical aspects in sleeve gastrectomy. Obes Surg 2007; 17:1534-5.

12. Petersen W, Meile T, Kuper M, Zdichavsky M, Konigsrainer A, Schneider J. Functional Importance of Laparoscopic Sleeve Gastrectomy for the Lower Esophageal Sphincter in Patients with Morbid Obesity. Obes Surg. 2012;22:360-6.

13. Chiu S, Birch D, Shi X, Sharma A, Karmali S. Effect of sleeve gastrectomy on gastroesophageal reflux disease: a systematic review. Surg Obes Relat Dis. 2011;7:510-5.

14. Boza C, Salinas J, Salgado N, Pérez G, Raddatz A, Funke R, et al. Laparoscopic sleeve gastrectomy as a Stand-Alone Procedure for morbid obesity: Report of 1000 cases and 3-year follow-up. Obes Surg. 2012;22:866-71.

15. Cottam D, Qureshi FG, Mattar SG, Sharma S, Holover $\mathrm{S}$, Bonanomi $\mathrm{G}$, et al. Laparoscopic sleeve gastrectomy as an initial weight loss procedure for high-risk patients with morbid obesity. Surg Endosc. 2006;20:859-63.

16. Gagner M, Deitel M, Kalberer TL, Erickson AL, Crosby RD. The Second International Consensus Summit for Sleeve Gastrectomy, March 19-21, 2009. Surg Obes Relat Dis. 2009;5:476-85.

17. Eubanks S, Edwards C, Fearing N, Ramaswamy A, De la Torre R, Thaler K, et al. Use of endoscopic stents to treat anastomotic complications after bariatric surgery. J Am Coll Surg. 2008;206:935-9.

18. Dapri G, Cadière G, Himpens J. Laparoscopic seromyotomy for long stenosis after sleeve gastrectomy with or without duodenal switch. Obes Surg. 2009;19:495-9.

19. Sudan R, Kasotakis G, Betof A, Wright A. Sleeve gastrectomy strictures: technique for robotic-assisted strictureplasty. Surg Obes Relat Dis. 2010;6:434-6.

20. Lacy A, Ibarzabal A, Pando E, Adelsdorfer C, Delitala A, Corcelles R, et al. Revisional surgery after sleeve gastrectomy. Surg Laparosc Endosc Percutan Tech. 2010;20:315-6.

21. Triantafyllidis G, Lazoura O, Sioka E, Tzovaras G, Antoniou A, Katerina V, et al. Anatomy and complications following laparoscopic sleeve gastrectomy: Radiological evaluation and imaging pitfalls. Obes Surg. 2011;21:473-8.

22. Spyropoulos C, Kehagias I, Panagiotopoulos S, Mead 
N, Kalfarentzos F. Revisional bariatric surgery: 13year experience from a tertiary institution. Arch Surg. 2010;145:173-7.

23. Victorzon M. Revisional bariatric surgery by conversion to gastric bypass or sleeve-good short term outcomes at higher risks. Obes Surg. 2012;22:29-33.
24. Deylgat B, D`Hondt M, Pottel H, Vansteenkiste F, Van Rooy R, Devriendt D. Indications, safety, and feasibility of conversion of failed bariatric surgery to Roux-en-Y gastric bypass: a retrospective comparative study with primary laparoscopic Roux-en Y gastric bypass. Surg Endosc. 2012;26:2997-3002. 